\title{
Insegnare il Settecento, a cura di Rosa Maria Loretelli e Alberto Postigliola
}

\section{Paola Sosso}

\section{Q OpenEdition}

1 Journals

\section{Edizione digitale}

URL: https://journals.openedition.org/studifrancesi/39367

DOI: 10.4000/studifrancesi.39367

ISSN: 2421-5856

\section{Editore}

Rosenberg \& Sellier

\section{Edizione cartacea}

Data di pubblicazione: 1 décembre 2004

Paginazione: 368-369

ISSN: 0039-2944

Notizia bibliografica digitale

Paola Sosso, «Insegnare il Settecento, a cura di Rosa Maria Loretelli e Alberto Postigliola», Studi

Francesi [Online], 143 (XLVIII | II) | 2004, online dal 30 novembre 2015, consultato il 19 mai 2021. URL: http://journals.openedition.org/studifrancesi/39367; DOI: https://doi.org/10.4000/studifrancesi. 39367

Questo documento è stato generato automaticamente il 19 mai 2021.

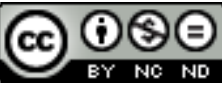

Studi Francesi è distribuita con Licenza Creative Commons Attribuzione - Non commerciale - Non opere derivate 4.0 Internazionale. 


\title{
Insegnare il Settecento, a cura di Rosa Maria Loretelli e Alberto Postigliola
}

\author{
Paola Sosso
}

\section{NOTIZIA}

Insegnare il Settecento, Atti del convegno della Società Italiana sul secolo XVIII, Roma, 7-9 maggio 1999, a cura di ROSA MARIA LORETELLI e ALBERTO POSTIGLIOLA, Itinerari, anno XLI, 3 , 2002, pp. 160.

$1 \quad$ II volume raccoglie gli atti relativi al convegno tenutosi a Roma dal 7 al 9 maggio 1999 organizzato dalla Società Italiana di studi sul secolo XVIII in collaborazione con il Dipartimento di Anglistica dell'Università «La Sapienza» di Roma. L'intento, come ben si evince dai lavori presentati, è quello di ricostruire il modo in cui oggi si raffigura, all'interno della scuola e dell'università, l'immagine del Settecento. Il testo si apre con l'intervento di Giuseppe RICUPERATI che riflette su La proposta del convegno: che cosa significa insegnare il Settecento nella scuola e nell'università (pp. 5-28): un ritorno al Settecento, spiega lo studioso, può innanzitutto aiutarci, soprattutto in Europa, a sviluppare un approccio comparatista allo studio della letteratura, perché fu proprio il Secolo dei Lumi a porsi il problema di una letteratura universale, slegata dall'idea di nazione. Un altro spazio di identificazione che il secolo dei philosophes può offrirci lo si trova nel concetto di «religione civile»(formulato per primo da Rousseau), utile in un momento storico come quello attuale in cui è necessario governare processi di assimilazione e ridare un senso a parole come libertà, democrazia e uguaglianza. Lo studioso non vuol certo fare del Siècle des Lumières un oggetto di utopia, ma piuttosto ricordare come il processo dell'Illuminismo si interruppe, ed è quindi necessario riprendere oggi una sfida che affonda le sue radici nello stesso terreno, e in questo anche gli errori commessi dai nostri predecessori ci possono essere utili. Jean MONDOT sottolinea, nell'articolo che segue (L 'enseignement du XVIIIe siècle en France, pp. 29-38) la specificità del diciottesimo secolo all'interno della storia francese, evidenziando come lo studio del Siècle des Lumières sia un pilier della scuola e dell'università. Più 
prettamente dedicato all'ambito scolastico è l'intervento di Dario GENERALI, I valori della ragione e prassi didattica. "Insegnare il Settecento» come progetto di riqualificazione dell'istituzione scolastica, pp. 39-60), che mette in evidenza gli aspetti che ancor oggi suscitano, o dovrebbero suscitare, l'interesse degli studenti della scuola superiore impegnati nello studio dell'Illuminismo: alcuni percorsi esemplificativi appaiono particolarmente interessanti se proposti alle classi con l'intento di riscoprire gli elementi qualificanti della modernità e ribadire la centralità della cultura nell'organizzazione scolastica. Dedicati ad ambiti specifici sono gli interventi di Cecilia CAMPA che analizza Il Settecento nell'insegnamento e nella ricerca musicologica (pp. 61-72), e quello di Maurizio MAMIANI, che si occupa dell'insegnamento della scienza nel '700 (Insegnare la scienza, pp. 73-80). Il saggio che segue, redatto Carlo BORGHERO presenta le differenti immagini della filosofia del Settecento apparse sulla scena storiografica negli ultimi decenni, concentrandosi in particolare sulla cultura filosofica italiana per mettere in luce alcune specificità del nostro paese (Immagini filosofiche dell'Illuminismo $e$ storia patria, pp. 81-105): prendendo le mosse dall'avversione degli ambienti tradizionalisti cattolici e dalla condanna di Croce e del neoidealismo, l'articolo arriva ad analizzare alcune posizioni più recenti (S. Landucci, A. Santucci, G. Benrekassa, per citarne solo alcuni) fino ad offrire un panorama ricco e variegato che permette di mettere in relazione immagini storiografiche della filosofia settecentesca e autorappresentazioni della storia culturale italiana. Il Settecento nella francesisitca italiana è il titolo dell'intervento di Simone CARPENTARI MESSINA, pp. 107-138), che, collegandosi e completando gli studi Lionello Sozzi del 1979 e quello di Franco Piva del 1990, illustra gli orientamenti della ricerca italiana sul Settecento francese dal 1990 al 2000. Dopo aver evidenziato la frammentazione e la mancanza di linee direttrici comuni, la studiosa sottolinea la presenza attiva e vivace di alcune riviste (Studi francesi, Micromegas, Franco-Italica) e l'importanza della Storia della civiltà letteraria francese curata da Lionello Sozzi (UTET, 1993) in cui, per la parte relativa al '700, appaiono alcuni tratti metodologici degni di rilievo: innanzitutto una periodizzazione lunga, che estende $i$ Lumi a monte, dal 1680 e a valle, fino ad includere i primi due decenni dell'Ottocento. Incompleto si presenta nella Storia della civiltà francese l'approccio socio-culturale, ma del resto una simile mancanza si rileva in gran parte della ricerca italiana, con alcune lodevoli eccezioni (fra gli altri vengono ricordati i lavori di B. Craveri, di E. Mannucci e di A. Basso ed alcuni studi sul teatro). La pluridisciplinarità è centrale anche nell'impegno di un gruppo di lavoro di Torino, presieduto da Lionello Sozzi, che mira a coinvolgere nelle sue attività non soltanto studiosi di discipline umanistiche, ma anche specialisti di altri settori. Studi su autori radicali o considerati fino ad ora minori, lavori su ombre e alterazioni dei lumi, pubblicazioni relative alle forme romanzesche (F. Piva, D. Gallingani, A. Pizzorusso fra gli altri), sui mémoires, sulle autobiografie (P. Toffano, L. Omacini, F. Bassani, B. Anglani...e ancora una volta, la nostra lista non può che essere incompleta) arricchiscono un panorama in cui sorprende il disinteresse per la poesia e la poca attenzione riservata al teatro. Anche nel settore della linguistica la ricerca individuale prevale sul lavoro collettivo: lo studio della lingua tende a privilegiare l'approccio lessicografico, anche se non mancano lavori sulla traduzione e sulla didattica. Autore prediletto dalla ricerca italiana permane Montesquieu, con numerosi italiani che partecipano all'edizione in corso delle Cuvres complètes; fervidi rimangono gli studi su Diderot (P. Quintili, M. Modica...), mentre la filosofia di Voltaire non sembra più destare un vivo interesse presso gli studiosi italiani; per Rousseau, gli studi si sono orientati verso i problemi esistenziali e le pulsioni dell'io. Anche se alcuni settori di 
punta della ricerca internazionale sono rimasti esclusi dalla riflessione di stampo italiano negli anni 1990-2000 (ad esempio i gender studies) o sono stati affrontati in modo parziale (utopia, intellettuali e potere, forme epistolari, letteratura clandestina...), appaiono nuove e degne di ampio sviluppo alcune problematiche finora rimaste secondarie, prime fra tutte le indagini sulle contraddizioni che animano la vita culturale del XVIII secolo: da qui, auspica la studiosa, possono partire nuovi progetti di respiro internazionale. Interdisciplinary Approches to the Eighteenth Century in Britain: the Luxury Project è il titolo dello studio di Maxime BERG p. 139-147), che precede l'ultimo intervento di Rosa Maria соцомво dedicato a Il Sapere delle donne: spazi dati e spazi contrattati nell'Inghilterra del Diciottesimo secolo (pp. 149-159). 